\title{
Split bounded extension algebras and Han's conjecture
}

\author{
Claude Cibils, Marcelo Lanzilotta, Eduardo N. Marcos, \\ and Andrea Solotar*
}

\begin{abstract}
A main purpose of this paper is to prove that the class of finite dimensional algebras which verify Han's conjecture is closed under split bounded extensions.
\end{abstract}

2010 MSC: 18G25, 16E40, 16E30, 18G15

Keywords: Hochschild, homology, relative, Han, quiver

\section{Introduction}

Given a finite dimensional algebra $A$ over an algebraically closed field $k$, Han's conjecture relates two homological invariants associated to $A$ : its global dimension and its Hochschild homology. In the commutative case - non necessarily finite dimensional but finitely generated - the finiteness of the global dimension is equivalent to the fact that $A$ is geometrically regular [3, 30]. In general we are going to say that an algebra with finite global dimension is smooth.

On the other hand, we consider Hochschild homology of $A$. Let $A^{e}=A \otimes A^{o p}$ be the enveloping algebra. Let us recall that given an $A$-bimodule $X$ - or equivalently a left or right $A^{e}$-module -, the Hochschild homology of $A$ with coefficients in $X$ is $H_{*}(A, X)=\operatorname{Tor}_{*}^{A^{e}}(A, X)$; it is functorial in both variables.

Han's conjecture [20] states that for $A$ finite dimensional, $A$ is smooth if and only if $H_{n}(A, A)=0$ for $n>>0$. The direct implication is true.

Next we recall some previous results. Well before being formulated, Han's conjecture has been proved for commutative algebras which are finitely generated, which encompasses finite dimensional commutative algebras, see [8, 4]. Y. Han proved the conjecture for monomial algebras in [20]. P.A. Bergh and D. Madsen have shown that it holds in characteristic zero for graded finite dimensional local algebras, Koszul algebras and graded cellular algebras [6]. They have also obtained a confirmation of Han's conjecture in [7] for trivial extensions of several sorts of algebras, by proving that their Hochschild homology is non zero in large enough degrees. P.A. Bergh and K. Erdmann proved in [5] that quantum complete intersections - at a non-root of unity - satisfy Han's conjecture, as well as A. Solotar

${ }^{*}$ This work has been supported by the projects UBACYT 20020130100533BA, PIPCONICET 11220150100483CO, USP-COFECUB. The third mentioned author was supported by the thematic project of FAPESP 2014/09310-5 and acknowledges support from the "Brazilian-French Network in Mathematics". The fourth mentioned author is a research member of CONICET (Argentina) and a Senior Associate at ICTP. 
and M. Vigué-Poirrier [32] for a generalisation of quantum complete intersections and for a family of algebras which are in some sense opposite to these last ones. Later, A. Solotar, M. Suárez-Alvarez and Q. Vivas proved in [31] Han's conjecture for quantum generalized Weyl algebras (out of a few exceptional cases). In [13] null-square projective algebras extensions were considered, the present paper goes further in this direction.

Concerning the commutative case, it is worth to mention that in characteristic zero, in positive degrees $H H_{n}(A)$ has a decomposition, called Hodge decomposition, as a direct sum of subspaces, see for example [18, 28, 33. One of them is the $n$-th exterior power of the $A$-module of Kähler differentials, $\Omega_{A \mid k}^{n}$ and another one is $D_{n}(A \mid k)$, the André-Quillen homology of the commutative $k$-algebra $A$. When $A$ is smooth, in positive degrees $H H_{n}(A)=\Omega_{A \mid k}^{n}$ and the other summands annihilate. In fact, the main condition for smoothness is that $D_{n}(A \mid k)=0$ for positive $n$ [23], and the Jacobi-Zariski long exact sequence for André-Quillen homology relating $D_{n}(A \mid k), D_{n}(A \mid B)$ and $D_{n}(B \mid k)$ for any extension of algebras $k \subseteq B \subseteq A$ plays an important role.

In the non commutative setting André-Quillen homology does not exist, but A. Kaygun has proved recently in [24, 25] the existence of a Jacobi-Zariski long exact sequence starting in degree one for Hochschild homology for any extension of $k$-algebras $B \subseteq A$, such that $A$ is $B$-flat. It relates the ordinary Hochschild homologies of $A$ and $B$ with the relative Hochschild homology of $A$ with respect to $B$. In this paper, with different hypotheses we also obtain a long exact sequence of Jacobi-Zariski type for large enough degrees.

We consider split extension algebras in relation with Han's conjecture. By definition, a split extension algebra over a field $k$ is a $k$-algebra of the form $A=B \oplus M$, where $B$ is a subalgebra of $A$ and $M$ is a two-sided ideal of $A$. As a consequence of our work, we prove that in some cases, adding or deleting arrows to a quiver - even adding or deleting certain relations - does not change the situation with respect to Han's conjecture, see also [14]. Indeed, these processes are special cases of split extension algebras, see Example [2.213. In a subsequent work, conditions will be given for these operations to fit within the framework of the theory we provide in this paper.

Next we describe the contents of this article. In Section 2 in order to compute the relative Hochschild (co)homology introduced by G. Hochschild in [22], we construct a reduced relative bar resolution of a split extension algebra. We use it particularly when $M$ is $B$-tensor nilpotent, that is if there exists $n$ such that $M^{\otimes_{B} n}=0$

In Section 3 we obtain a Jacobi-Zariski long exact sequence in the following situation. A $B$-bimodule $M$ is called bounded if $M$ is $B$-tensor nilpotent, of finite projective dimension as $B$-bimodule and projective either as left or as right $B$ module. A split bounded extension algebra is a split extension $A=B \oplus M$ where $M$ is bounded. For these algebras we obtain a Jacobi-Zariski long exact sequence in large enough degrees. We set up techniques based on nearly exact sequences of complexes, see Definition 3.1. Actually the relative resolution of Section 2 provides a nearly exact sequence, which in turn gives the required Jacobi-Zariski long exact sequence in large enough degrees.

In Section 4 we prove our main result: the class $\mathcal{H}$ of finite dimensional algebras which verify Han's conjecture is closed under split bounded extensions. More precisely if $A=B \oplus M$ is such an extension, then $A \in \mathcal{H}$ if and only if $B \in \mathcal{H}$. We 
point out that this result does not depend on the associative structure of $M$, but on properties of its $B$-bimodule structure, see Definitions 3.4 and 3.6 The proofs make use of the Jacobi-Zariski long exact sequence, and of the reduced relative bar resolution.

\section{A reduced relative bar resolution for split extension al- gebras}

Let $B \subset A$ be an extension of algebras over a field $k$. In this context $\mathrm{G}$. Hochschild introduced in 22] relative homological algebra, which corresponds to consider the exact category of $A$-modules with respect to $B$-split short exact sequences, see [27. 9]. More precisely, an induced module is an $A$-module of the form $A \otimes_{B} M$, where $M$ is a left $B$-module. An $A$-module $P$ is relative projective if any $A$ morphism $X \rightarrow P$ which has a $B$-section has an $A$-section. Equivalently, $P$ is relative projective if it is an $A$-direct summand of an induced module. There are enough relative projectives since for any $A$-module $X$ the canonical $A$-map $A \otimes_{B}$ $X \rightarrow X$ has a $B$-section. Of course if $B=k$ we recover the ordinary definition, and if $B=A$ all modules are relative projective.

A relative projective resolution of an $A$-module $X$ is a sequence

$$
\cdots \stackrel{d}{\rightarrow} P_{2} \stackrel{d}{\rightarrow} P_{1} \stackrel{d}{\rightarrow} P_{0} \rightarrow X \rightarrow 0
$$

where each $P_{i}$ is a relative projective $A$-module, the $d^{\prime}$ 's are $A$-morphisms, $d^{2}=0$ and there exists a $B$-contracting homotopy, see [22, p. 250].

Two relative projective resolutions of $X$ are homotopic and the functor $A \otimes_{B}-$ is exact, so that for $X$ and $Y$ respectively right and left $A$-modules, the functor $\operatorname{Tor}_{*}^{A \mid B}(X, Y)$ is well defined. For $X$ and $Y$ left $A$-modules, the functor $\operatorname{Ext}_{A \mid B}^{*}(X, Y)$ is well defined.

Consider the extension of enveloping algebras $B^{e} \subset A^{e}$. For $X$ an $A$-bimodule, the relative Hochschild homology and cohomology vector spaces are defined in [22] respectively as follows:

$$
H_{*}(A \mid B, X)=\operatorname{Tor}_{*}^{A^{e} \mid B^{e}}(X, A) \text { and } H^{*}(A \mid B, X)=\operatorname{Ext}_{A^{e} \mid B^{e}}^{*}(A, X) .
$$

Observe that in 22] those vector spaces are defined with respect to the extension $B \otimes A^{o p} \subset A^{e}$. This turns out to be equivalent since the relative canonical resolution of $A$ is relative projective in both situations, and the canonical contracting homotopies agree.

Being derived functors, they can be computed using an arbitrary relative projective resolution. In particular these vector spaces are the homology and the cohomology of the following chains and cochains complexes $C_{*}(A \mid B, X)$ and $C^{*}(A \mid B, X)$ :

$$
\begin{aligned}
& \cdots \stackrel{b}{\rightarrow} X \otimes_{B^{e}} A^{\otimes_{B} n} \stackrel{b}{\rightarrow} \cdots \stackrel{b}{\rightarrow} X \otimes_{B^{e}} A \stackrel{b}{\rightarrow} X_{B} \rightarrow 0, \\
0 \rightarrow & X^{B} \stackrel{b}{\rightarrow} \operatorname{Hom}_{B^{e}}(A, X) \stackrel{b}{\rightarrow} \cdots \stackrel{b}{\rightarrow} \operatorname{Hom}_{B^{e}}\left(A^{\otimes_{B} n}, X\right) \stackrel{b}{\rightarrow} \cdots
\end{aligned}
$$

where

$$
\begin{gathered}
X_{B}=X \otimes_{B^{e}} B=X /\langle b x-x b\rangle=H_{0}(B, X), \\
X^{B}=\operatorname{Hom}_{B^{e}}(B, X)=\{x \in X \mid b x=x b \text { for all } b \in B\}=H^{0}(B, X),
\end{gathered}
$$

and where the formulas for the boundaries and coboundaries are the ordinary ones. 
Definition 2.1 An extension of algebras $B \subset A$ is split if there is a morphism of algebras $\pi: A \rightarrow B$ which is a retraction to the inclusion, that is $\pi(b)=b$ for all $b \in B$.

Clearly $B \subset A$ is split if and only if there exists a two-sided ideal $M$ of $A$ such that $A=B \oplus M$.

Next we provide some examples of split extensions. In the last example we add arrows to the quiver of a bound quiver algebra. Note that in relation to the finitistic dimension conjecture, E.L. Green, C. Psaroudakis and $\varnothing$. Solberg [19] have considered the case of adding exactly one arrow, which leads to a trivial extension.

\section{Examples 2.2}

1. Let $B$ be an algebra, let $N$ be a $B$-bimodule and let $T$ be the tensor algebra

$$
T=T_{B}(N)=B \oplus N \oplus N \otimes_{B} N \oplus \cdots .
$$

Let $T^{>i}=N^{\otimes_{B} i+1} \oplus N^{\otimes_{B} i+2} \oplus \cdots$

We have $T=B \oplus T^{>0}$, that is $T$ is a split extension. Moreover, if $J \subset T^{>0}$ is a two-sided ideal of $T$, then $B \subset T / J$ is a split extension as well.

2. Let $Q$ be a finite quiver, that is $Q=\left(Q_{0}, Q_{1}, s, t\right)$ where $Q_{0}$ and $Q_{1}$ are finite sets called respectively vertices and arrows, and $s, t: Q_{0} \rightarrow Q_{1}$ are maps called respectively source and target. Let $A=k Q / I$ be a bound quiver algebra, where $k Q$ is the path algebra of $Q$ and $I$ is an admissible two-sided ideal of $k Q$, see [15, 16, 17] and [1, 29]. The extension $B=k Q_{0} \subset A$ is split.

3. Let $B=k Q / I$ be a bound quiver algebra, and let $F$ be a finite set of new arrows, that is $F$ is a finite set with two maps $s, t: F \rightarrow Q_{0}$. Let $Q_{F}$ be the quiver with the same vertices than $Q$, while its arrows are $Q_{1} \sqcup F$.

Let $B_{F}=k Q_{F} /\langle I\rangle_{k Q_{F}}$, where the denominator is the two-sided ideal of $k Q_{F}$ generated by $I$. It is easily proven that $B_{F}=T_{B}(N)$ where

$$
N=\bigoplus_{a \in F} B t(a) \otimes s(a) B .
$$

Let $J \subset B_{F}^{>0}$ be a two-sided ideal of $B_{F}$. The algebra

$$
A=B_{F} / J=B \oplus\left(B_{F}^{>0} / J\right)
$$

is also a split extension.

The first item of the next result is a generalisation of a reduced bar resolution obtained in [11, Lemma 2.1].

Theorem 2.3 Let $A=B \oplus M$ be a split extension of algebras.

1. There is a reduced relative bar resolution of $A$ as $A$-bimodule

$$
\cdots \stackrel{d}{\rightarrow} A \otimes_{B} M^{\otimes_{B} n} \otimes_{B} A \stackrel{d}{\rightarrow} \cdots \stackrel{d}{\rightarrow} A \otimes_{B} M \otimes_{B} A \stackrel{d}{\rightarrow} A \otimes_{B} A \stackrel{d}{\rightarrow} A \rightarrow 0
$$

where the formulas for the d's are those of the ordinary bar resolution, see [21, 22]. 
In what follows the formulas for the (co)boundaries are the ordinary ones.

2. Let $X$ be an A-bimodule. The homology of the following chain complex $C_{*}^{M}(A \mid B, X)$ is $H_{*}(A \mid B, X)$.

$$
C_{*}^{M}(A \mid B, X): \quad \cdots \stackrel{b}{\rightarrow} X \otimes_{B^{e}} M^{\otimes_{B} n} \stackrel{b}{\rightarrow} \cdots \stackrel{b}{\rightarrow} X \otimes_{B^{e}} M \stackrel{b}{\rightarrow} X_{B} \rightarrow 0
$$

3. The cohomology of the following cochain complex $C_{M}^{*}(A \mid B, X)$ is $H^{*}(A \mid B, X)$.

$$
0 \rightarrow X^{B} \stackrel{b}{\rightarrow} \operatorname{Hom}_{B^{e}}(M, X) \stackrel{b}{\rightarrow} \cdots \stackrel{b}{\rightarrow} \operatorname{Hom}_{B^{e}}\left(M^{\otimes_{B} n}, X\right) \stackrel{b}{\rightarrow} \cdots
$$

Proof. By construction, the bimodules involved in the first item are induced bimodules, hence they are relative projective. Let $a=a_{B}+a_{M}$ be the decomposition of $a \in A=B \oplus M$, and let

$$
t\left(a_{1} \otimes m_{2} \otimes \cdots \otimes m_{n+1} \otimes a_{n+2}\right)=1 \otimes\left(a_{1}\right)_{M} \otimes m_{2} \otimes \cdots \otimes m_{n+1} \otimes a_{n+2} .
$$

It is easily proven that $t$ is a well defined $B^{e}$-morphism, which is a contracting homotopy.

The second item is obtained by applying the functor $X \otimes_{A^{e}}-$ to the resolution, and the following canonical isomorphism where $Z$ is a $B$-bimodule

$$
X \otimes_{A^{e}}\left(A \otimes_{B} Z \otimes_{B} A\right)=X \otimes_{B^{e}} Z .
$$

The last item is obtained analogously.

Remark 2.4 For later use, we record that the contracting homotopy $t$ in the previous proof is also a right $A$-module map.

A $B$-bimodule $M$ is $B$-tensor nilpotent if there exists $n$ such that $M^{\otimes_{B} n}=0$. Moreover $n$ is the index of $B$-tensor nilpotency if $M^{\otimes_{B} n-1} \neq 0$. For instance, let $k Q$ be the path algebra of a quiver $Q$. The $k Q_{0}$-bimodule $\left\langle Q_{1}\right\rangle \subset k Q$ is $k Q_{0}$-tensor nilpotent if and only if there is no oriented cycle in $Q$.

Corollary 2.5 Let $A=B \oplus M$ be a split extension, where $M$ is $B$-tensor nilpotent of index $n$. Let $X$ be an $A$-bimodule. For $* \geq n$ we have

$$
H_{*}(A \mid B, X)=0 \text { and } H^{*}(A \mid B, X)=0 .
$$

Let $C_{*}(A, X)$ be the ordinary chain complex

$$
C_{*}(A, X): \cdots \stackrel{b}{\rightarrow} X \otimes A^{\otimes n} \stackrel{b}{\rightarrow} \cdots \stackrel{b}{\rightarrow} X \otimes A \stackrel{b}{\rightarrow} X \rightarrow 0
$$

whose homology is the Hochschild homology $H_{*}(A, X)$ of an $A$-bimodule $X$. Towards obtaining a Jacobi-Zariski long exact sequence for a split extension algebra, we observe the following.

Proposition 2.6 Let $A=B \oplus M$ be a split extension of algebras, and let $X$ be an $A$-bimodule. For $* \geq 1$, there is a sequence of chain complexes

$$
0 \rightarrow C_{*}(B, X) \stackrel{\iota}{\rightarrow} C_{*}(A, X) \stackrel{\kappa}{\rightarrow} C_{*}^{M}(A \mid B, X) \rightarrow 0
$$

where $\iota$ is injective, $\kappa$ is surjective and $\kappa \iota=0$.

In degree 0 we have the sequence

$$
0 \rightarrow X \stackrel{1}{\rightarrow} X \rightarrow X_{B} \rightarrow 0 .
$$


Proof. The definition of the map $\iota$ is clear, and it is also clear that $\iota$ is an injective map of complexes. The map $\kappa$ given by

$$
x \otimes a_{1} \otimes \cdots \otimes a_{n} \mapsto x \otimes_{B^{e}}\left[\left(a_{1}\right)_{M} \otimes_{B} \cdots \otimes_{B}\left(a_{n}\right)_{M}\right]
$$

is surjective, and $\kappa \iota=0$. The verification that $\kappa$ is a map of complexes does not raise any difficulty. It uses extensively that $\left(a a^{\prime}\right)_{M}=a_{M} a_{M}^{\prime}+a_{B} a_{M}^{\prime}+a_{M} a_{B}^{\prime}$ for $a, a^{\prime} \in A$ and that the first tensor product in $C_{*}^{M}(A \mid B, X)$ is over $B^{e}$.

Remark 2.7 Considering $C_{*}(A \mid B, X)$ instead of $C_{*}^{M}(A \mid B, X)$, and $\kappa^{\prime}$ given by

$$
x \otimes a_{1} \otimes \cdots \otimes a_{n} \mapsto x \otimes_{B^{e}}\left[a_{1} \otimes_{B} \cdots \otimes_{B} a_{n}\right]
$$

does not give in general $\kappa^{\prime} \iota=0$.

Let $A=B \oplus M$ be a split extension. In the ensuing decomposition of the vector space $A^{\otimes n}$, let $\left[M_{p} B_{q}\right]$ be the direct sum of the direct summands containing $p$ tensorands in $M$ and $q$ tensorands in $B$, with $p+q=n$. For instance - omitting the $\otimes$ signs - we have that

$$
\left[M_{2} B_{2}\right]=M M B B \oplus M B M B \oplus M B B M \oplus B M B M \oplus B M M B \oplus B B M M
$$

which is a direct summand of $A^{\otimes 4}$.

We set

$$
K_{n, 0}=\operatorname{Ker}\left(X \otimes M^{\otimes n} \rightarrow X \otimes_{B^{e}} M^{\otimes_{B} n}\right) .
$$

Lemma 2.8 In the situation of Proposition 2.6

$$
\begin{aligned}
& \text { Ker } \kappa=\bigoplus_{\substack{p+q=n \\
p \geq 0}, 0} X \otimes\left[M_{p} B_{q}\right] \oplus K_{n, 0} \\
& \operatorname{Im} \iota=X \otimes\left[M_{0} B_{n}\right] \\
& \operatorname{Ker} \kappa / \operatorname{Im} \iota=\bigoplus_{\substack{p+q=n \\
p>0}>0} X \otimes\left[M_{p} B_{q}\right] \oplus K_{n, 0} .
\end{aligned}
$$

Proof. Consider the direct sum decomposition

$$
X \otimes A^{\otimes n}=\bigoplus_{\substack{p+q=n \\ p \geq 0 \\ q \geq 0}} X \otimes\left[M_{p} B_{q}\right] .
$$

If $q>0$, then $\kappa\left(X \otimes\left[M_{p} B_{q}\right]\right)=0$, hence

$$
\bigoplus_{\substack{p+q=n \\ p \geq 0 \\ q>0}} X \otimes\left[M_{p} B_{q}\right] \subset \mathrm{Ker} \kappa .
$$

Instead if $q=0$, then $\kappa_{X \otimes M^{\otimes n}}$ is not zero in general and its kernel is denoted $K_{n, 0}$. It follows that Ker $\kappa$ is as stated.

In turn, in the above direct sum decomposition of $X \otimes A^{\otimes n}$, the direct summand for $p=0$ and $q=n$ is clearly $\operatorname{Im} \iota$. This vector space is one of the direct summands obtained above for Ker $\kappa$. The decomposition of $\operatorname{Ker} \kappa / \operatorname{Im} \iota$ follows. 


\section{Nearly exact sequences and the Jacobi-Zariski long ex- act sequence}

In this section we will prove that if a sequence as (2.6) has zero homology for large enough degrees at the second page of the associated spectral sequence, then there is a long exact sequence in homology starting at this precise degree.

Definition 3.1 A sequence of chain complexes concentrated in non negative degrees

$$
0 \rightarrow C_{*} \stackrel{\iota}{\rightarrow} D_{*} \stackrel{\kappa}{\rightarrow} E_{*} \rightarrow 0
$$

is $m$-nearly exact if

- $\iota$ is injective,

- $\kappa$ is surjective,

$-\kappa \iota=0$

- the chain complex $\mathrm{Ker} \kappa / \mathrm{Im} \iota$ with boundary induced by the boundary of $D$, is exact in degrees $\geq m$.

We will prove later on that under some hypotheses, the sequence of Proposition 2.6 is nearly exact.

Theorem 3.2 Let

$$
0 \rightarrow C_{*} \stackrel{\iota}{\rightarrow} D_{*} \stackrel{\kappa}{\rightarrow} E_{*} \rightarrow 0
$$

be a m-nearly exact sequence of chain complexes. There is a long exact sequence as follows:

$$
\ldots \stackrel{\delta}{\rightarrow} H_{m+1}(C) \stackrel{\iota}{\rightarrow} H_{m+1}(D) \stackrel{\kappa}{\rightarrow} H_{m+1}(E) \stackrel{\delta}{\rightarrow} H_{m}(C) \stackrel{\iota}{\rightarrow} H_{m}(D) .
$$

Proof. We will use standard results on spectral sequences, see for instance [26] or [34].

The homological double complex arising from the sequence (3.1) with the standard change of signs, has the complexes $E, D$ and $C$ at columns $p=0,1$ and 2 respectively. Firstly we claim that this complex has zero homology in total degrees $\geq m+1$. Indeed, consider the spectral sequence given by the filtration by the rows. At the first page the columns corresponding to $p=0,2$ are zero since $\iota$ is injective and $\kappa$ is surjective. At column $p=1$ we have the homology vector spaces of the sequence (3.1) corresponding to the complex in the middle. Since the sequence is $m$-nearly exact, at the second page the column $p=1$ has zeros in degrees $\geq m$, and zeros elsewhere. This proves the claim.

Secondly we consider the filtration by columns. In page 1 of the corresponding spectral sequence, let $\iota_{1}$ and $\kappa_{1}$ be the horizontal maps induced by $\iota$ and $\kappa$ at the homology level of the complexes of the sequence (3.1). They are the morphisms of the intended long exact sequence. We assert that in degrees $\geq m+1$ there is exactness at the column corresponding to the homology of $D$. Indeed, the vector spaces at the second page at column $p=1$ are $\operatorname{Ker} \kappa_{1} / \operatorname{Im} \iota_{1}$. At these spots the differentials $d_{2}$ come from zero and go to zero. Hence these vector spaces live forever in the subsequent pages of the spectral sequence. We proved before that 
the complex has no homology in total degrees $\geq m+1$, hence these vector spaces vanish in degrees $\geq m+1$.

Finally we turn to the connecting homomorphism $\delta$. In the second page of the spectral sequence just considered, at columns $p=0,2$ we have respectively Coker $\kappa_{1}$ and Ker $\iota_{1}$. We assert that the differentials $d_{2}:$ Ker $\iota_{1} \rightarrow$ Coker $\kappa_{1}$ from total degree $m+1$ to total degree $m$ are isomorphisms, as well as in larger degrees. Indeed, in these degrees $\operatorname{Ker} d_{2}$ and Coker $d_{2}$ live forever in the spectral sequence, hence they vanish by the same argument than above. We assert that composing $d_{2}^{-1}$ with the inclusion of Ker $\iota_{1}$ and the canonical projection to Coker $\kappa_{1}$ provides the required connecting homomorphism $\delta$ of the long exact sequence for these degrees. Indeed, by construction $\operatorname{Ker} \delta=\operatorname{Im} \kappa_{1}$ and $\operatorname{Im} \delta=\operatorname{Ker} \iota_{1}$.

For the next result we assume that the $B$-bimodule $M$ verifies that for $*>0$ we have $\operatorname{Tor}_{*}^{B}\left(M, M^{\otimes_{B} n}\right)=0$ for all $n$. Note that this is fulfilled if $M$ is either a left or a right projective $B$-module.

Proposition 3.3 Let $A=B \oplus M$ be a split algebra, let $X$ be an A-bimodule and consider the sequence (2.6) as a double complex after performing the standard change of signs. Let $E_{1, *}^{2}$ be the second page of the spectral sequence obtained by filtering by rows.

There is a double complex $C_{*, *}$ which total homology is $E_{1, *}^{2}$. The filtration by columns of $C_{*, *}$ yields a spectral sequence. If $M$ verifies that for $*>0$ we have $\operatorname{Tor}_{*}^{B}\left(M, M^{\otimes_{B} n}\right)=0$ for all $n$, the terms at page 1 are

$$
F_{p, q}^{1}=\operatorname{Tor}_{p+q}^{B^{e}}\left(X, M^{\otimes_{B} p}\right) \text { for } p, q>0
$$

and 0 otherwise.

Proof. By Lemma 2.8

$$
E_{1, n}^{1}=\bigoplus_{\substack{p+q=n \\ p>0 \\ p>0}} X \otimes\left[M_{p} B_{q}\right] \oplus K_{n, 0} .
$$

The differential of this column is deduced from the one of $C_{*}(A, X)$. Clearly this column is the total complex of the double chain complex.

- $C_{p, q}=X \otimes\left[M_{p} B_{q}\right]$ for $p, q>0$,

- $C_{p, 0}=K_{p, 0}$ for $p>0$,

- 0 at other spots.

We modify momentarily $C_{*, *}$ at its bottom line as follows:

$$
C_{*, *}^{\prime}=X \otimes\left[M_{p} B_{q}\right] \text { for } p>0, q \geq 0 \text {, and } 0 \text { at other spots, }
$$

with differentials still inherited from $C_{*}(A, X)$.

We assert that the homology of the column $p=1$ of $C_{*, *}^{\prime}$ is $\operatorname{Tor}_{*}^{B^{e}}(X, M)$. To this purpose, we next recall a specific projective resolution of $M$ as a $B^{e}$-module which is provided in the proof of Proposition 4.1 of [12]. We have that the functor $X \otimes_{B^{e}}-$ applied to it yields the mentioned column, proving this way the assertion. As before, we omit the tensor product sign $\otimes$ over $k$.

Let

$$
{ }^{q} M^{p}=\underbrace{B \cdots B}_{q} M \underbrace{B \cdots B}_{p}
$$


and consider the following complex of free $B^{e}$-modules:

$$
\cdots \stackrel{d}{\rightarrow} \bigoplus_{\substack{p+q=n+1 \\ p>0}}{ }^{q} M^{p} \stackrel{d}{\rightarrow} \cdots \stackrel{d}{\rightarrow}{ }^{1} M^{2} \oplus^{2} M^{1} \stackrel{d}{\rightarrow}{ }^{1} M^{1} \stackrel{d}{\rightarrow} M \rightarrow 0,
$$

where the first differential is special, namely $d\left(b \otimes m \otimes b^{\prime}\right)=b m b^{\prime}$. In larger degrees, the differential is the differential of the total complex of the double complex which has ${ }^{q} M^{p}$ at the spot $(q, p)$, with vertical and horizontal differentials ${ }^{q} M^{p} \rightarrow{ }^{q} M^{p-1}$ and ${ }^{q} M^{p} \rightarrow{ }^{q-1} M^{p}$ given respectively by

$b_{1} \otimes \cdots \otimes b_{q} \otimes m \otimes b_{1}^{\prime} \otimes \cdots \otimes b_{p}^{\prime} \mapsto$ $(-1)^{q+1}\left[b_{1} \otimes \cdots \otimes b_{q} \otimes m b_{1}^{\prime} \otimes \cdots \otimes b_{p}^{\prime}+\right.$ $\left.\sum(-1)^{i} b_{1} \otimes \cdots \otimes b_{q} \otimes m \otimes b_{1}^{\prime} \otimes \cdots \otimes b_{i}^{\prime} b_{i+1} \otimes \cdots \otimes b_{p}^{\prime}\right]$

and

$b_{1} \otimes \cdots \otimes b_{q} \otimes m \otimes b_{1}^{\prime} \otimes \cdots \otimes b_{p}^{\prime} \mapsto$ $\sum(-1)^{i} b_{1} \otimes \cdots \otimes b_{i} b_{i+1} \otimes \cdots \otimes b_{q} \otimes m \otimes b_{1}^{\prime} \otimes \cdots \otimes b_{p}^{\prime}+$ $(-1)^{q} b_{1} \otimes \cdots \otimes b_{q} m \otimes b_{1}^{\prime} \otimes \cdots \otimes b_{p}^{\prime}$

We make precise that the vertical and horizontal differentials ${ }^{q} M^{1} \rightarrow{ }^{q} M^{0}$ and ${ }^{1} M^{p} \rightarrow{ }^{0} M^{p}$ are given respectively by

$b_{1} \otimes \cdots \otimes b_{q} \otimes m \otimes b_{1}^{\prime} \mapsto(-1)^{q+1} b_{1} \otimes \cdots \otimes b_{q} \otimes m b_{1}^{\prime}$

and

$b_{1} \otimes m \otimes b_{1}^{\prime} \otimes \cdots \otimes b_{p}^{\prime} \mapsto-b_{1} m \otimes b_{1}^{\prime} \otimes \cdots \otimes b_{p}^{\prime}$.

For the column $p=2$ of $C_{*, *}^{\prime}$, let $F_{\bullet} \rightarrow M$ be the previous projective resolution of $M$ by free bimodules. Its tensor product over $B$ with the left bar resolution

$$
\cdots \rightarrow B B M \rightarrow B M \rightarrow M \rightarrow 0
$$

of $M$ provides the following double complex $\mathbf{D}$ :

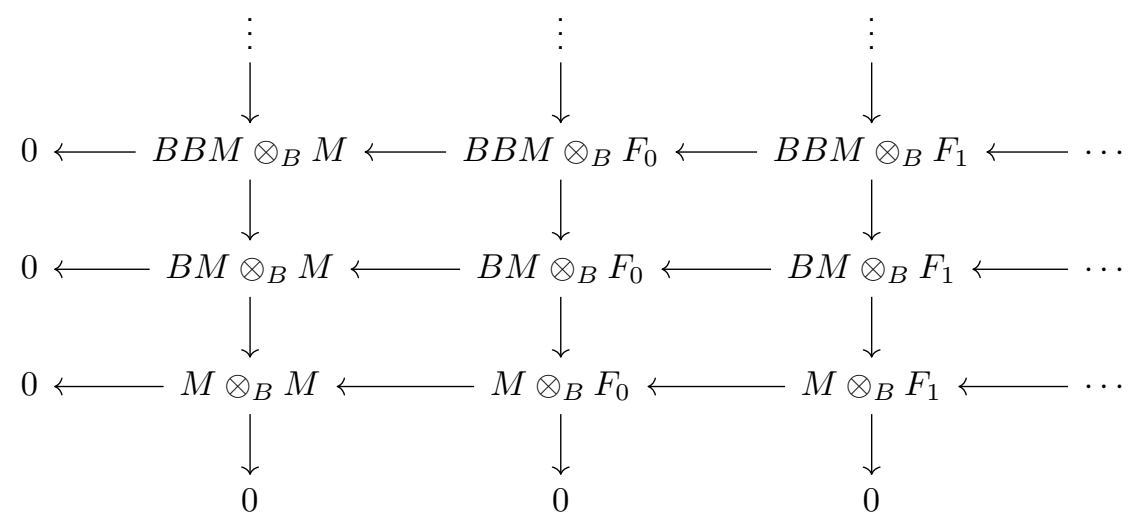

The bar resolution has a right $B$-module contracting homotopy. Hence the columns of $\mathbf{D}$ are acyclic and its total complex $\operatorname{Tot}(\mathbf{D})$ is exact.

However the bimodules of the bottom row and of the left column are not projective in general, while the others are. In order to obtain the required projective resolution of $M \otimes_{B} M$ as a bimodule, we proceed as in [12]. Let $\mathbf{S}$ be the double subcomplex of $\mathbf{D}$ given by the bottom row and the left column. We claim that $\operatorname{Tot}(\mathbf{D} / \mathbf{S}) \rightarrow M \otimes_{B} M$ is a free resolution of $M \otimes_{B} M$ as a $B$-bimodule. 
First note that the homology of the bottom row is precisely $\operatorname{Tor}_{*}^{B}(M, M)$, which is zero in positive degrees by hypothesis. Hence $\operatorname{Tot}(\mathbf{S})$ is exact in positive degrees, while in degree zero its homology is $M \otimes_{B} M$ : indeed, observe that for surjective morphisms $f: Y \rightarrow X$ and $g: Z \rightarrow X$, and $(f, g): Y \oplus Z \rightarrow X$ we have that

$$
\frac{\operatorname{Ker}(f, g)}{\operatorname{Ker} f \oplus \operatorname{Ker} g} \text { is isomorphic to } X
$$

by the map induced by $f$ or by $g$.

Next we consider the long exact sequence associated to the exact sequence of complexes

$$
0 \rightarrow \mathbf{S} \rightarrow \mathbf{D} \rightarrow \mathbf{D} / \mathbf{S} \rightarrow 0
$$

It shows that $\operatorname{Tot}(\mathbf{D} / \mathbf{S})$ is acyclic except in its last term where the homology is $M \otimes_{B} M$. This provides the required resolution of $M \otimes_{B} M$.

Next we iterate the process by tensoring the last resolution with the left bar resolution of $M$. As before, we use that $\operatorname{Tor}_{*}^{B}\left(M, M \otimes_{B} M\right)=0$ in positive degrees to infer a projective resolution of the $B$-bimodule $M \otimes_{B} M \otimes_{B} M$.

This shows that the homology of the $p$-th column is $\operatorname{Tor}_{*}^{B^{e}}\left(X, M^{\otimes_{B} p}\right)$.

In order to return to $C_{*, *}$, note that by (2.7) we have

$$
\left(X \otimes M^{\otimes p}\right) / K_{p, 0}=X \otimes_{B^{e}} M^{\otimes_{B} p}=\operatorname{Tor}_{0}^{B^{e}}\left(X, M^{\otimes_{B} p}\right) .
$$

Hence replacing the bottom row of $C^{\prime}$ by $K_{*, 0}$ yields surjective maps at the bottom stage of each column, therefore we have zero homology at spots of the bottom row of $C$.

Next we provide sufficient conditions to ensure that the sequence (2.6) of Proposition 2.6 is nearly exact.

Definition 3.4 Let $B$ be an algebra. $A$ B-bimodule $M$ is bounded if

- $M$ is B-tensor nilpotent,

- $M$ is of finite projective dimension as a $B^{e}$-module,

- $M$ is either a left or a right projective B-module.

Remark 3.5 Let $B$ be an algebra, and let $M$ be a $B$-bimodule with a $B$-associative structure, that is an associative map of $B$-bimodules $M \otimes_{B} M \rightarrow M$. Then $B \oplus M$ is a split extension algebra. Of course all split extensions occurs this way.

We underline that in the requirement that $M$ is bounded, the B-associative structure of $M$ is not involved.

Definition 3.6 $A$ split bounded extension $B \oplus M$ is a split extension where $M$ is bounded.

Proposition 3.7 Let $A=B \oplus M$ be a split bounded extension. Let $n$ be the index of $B$-tensor nilpotency of $M$. Let $u$ be the projective dimension of $M$ as a $B^{e}$-module, and let $X$ be an A-bimodule.

The sequence (2.6) is nu-nearly exact. 
Proof. We consider the spaces $F_{p, q}^{1}=\operatorname{Tor}_{p+q}^{B^{e}}\left(M^{\otimes_{B} p}, X\right)$ for $p, q>0$ of Proposition 3.3. On the one hand $F_{p, q}^{1}=0$ for $p \geq v$.

On the other hand, from [10, Chapter IX, Proposition 2.6] we infer that since $M$ is projective either as left or as right $B$-module, and is of projective dimension $u$ as a $B$-bimodule, then $M^{\otimes_{B} p}$ is of projective dimension $\leq p u$. Hence if $p+q \geq p u$, then $F_{p, q}^{1}=0$.

As a consequence, if $p+q \geq n u$, then $F_{p, q}^{1}=0$. By Proposition 3.3 we obtain that if $* \geq n u$ then $E_{1, *}^{2}=0$, which means that the column of homologies from the middle of the sequence (2.6) has in turn no homology in degrees $\geq n u$, that is the sequence is $n u$-nearly exact.

The previous result and Theorem 3.2 prove the following.

Theorem 3.8 Let $A=B \oplus M$ be a split bounded extension as in Proposition 3.7 . and let $X$ be an A-bimodule. There is a Jacobi-Zariski long exact sequence as follows.

$$
\begin{gathered}
\ldots \stackrel{\delta}{\rightarrow} H_{n u+1} m(B, X) \stackrel{\iota}{\rightarrow} H_{n u+1}(A, X) \stackrel{\kappa}{\rightarrow} H_{n u+1}(A \mid B, X) \stackrel{\delta}{\rightarrow} \\
\quad H_{n u}(B, X) \stackrel{\iota}{\rightarrow} H_{n u}(A, X) .
\end{gathered}
$$

\section{Han's conjecture}

A finite dimensional algebra is called smooth if it is of finite global dimension. As it is mentioned in the Introduction, the word smooth is originated in commutative algebra and is useful here for brevity. Note that for noetherian rings, the left and right global dimensions are equal, see [2].

Han's conjecture states that for $A$ a finite dimensional algebra, $H_{*}(A, A)$ vanishes in large enough degrees if and only if $A$ is smooth. Let $\mathcal{H}$ be the class of finite dimensional algebras which verify Han's conjecture. Our aim is to prove the following.

Theorem 4.1 Let $A=B \oplus M$ be a split bounded extension of finite dimensional algebras.

$A \in \mathcal{H}$ if and only if $B \in \mathcal{H}$.

The proof relies on the next result.

Proposition 4.2 Let $A=B \oplus M$ be a split bounded extension of finite dimensional algebras.

1. $H_{*}(A, A)$ vanishes in large enough degrees if and only if $H_{*}(B, B)$ vanishes in large enough degrees.

2. $A$ is smooth if and only if $B$ is smooth.

Remark 4.3 For a split extension $A=B \oplus M$, it is trivial that if $H_{*}(A, A)$ vanishes in large enough degrees, then the same happens for $H_{*}(B, B)$. Indeed, $H_{*}(-,-)$ is a functor on the category of algebras. Hence $H_{*}(B, B)$ is a direct summand of $H_{*}(A, A)$.

\section{Proof.}


1. Recall that $n$ is the index of $B$-nilpotency of $M$, and $u$ is the projective dimension of $M$ as a $B^{e}$-module. We claim that $H_{*}(A, A)$ and $H_{*}(B, B)$ are isomorphic if $* \geq n u+1$.

Recall that by Corollary 2.5 we have that $H_{*}(A \mid B, A)$ vanishes for $* \geq n$. Hence the Jacobi-Zariski long exact sequence of Theorem 3.8 shows that $H_{*}(B, A)$ and $H_{*}(A, A)$ are isomorphic for $* \geq n u+1$.

On the other hand $H_{*}(B, A)=H_{*}(B, B) \oplus H_{*}(B, M)$. Moreover,

$$
H_{*}(B, M)=\operatorname{Tor}_{*}^{B^{e}}(B, M) \text {. }
$$

Hence if $* \geq u$, then $H_{*}(B, M)=0$ and $H_{*}(B, A)=H_{*}(B, B)$.

2. The bimodule $M$ is projective from at least one side, we will suppose that $M$ is right projective. First we prove that if $A$ is smooth then $B$ is smooth. Let $\pi: A \rightarrow B$ be the retraction algebra map of $B \subset A$, with kernel $M$. Let $Y$ be a right $B$-module, and let $\bar{Y}$ be the $A$-module obtained by restricting scalars through $\pi$. We have $Y=\bar{Y}$ as right $B$-modules and $\bar{Y} M=0$.

Let $P_{*} \rightarrow \bar{Y}$ be a finite right $A$-projective resolution of $\bar{Y}$. It remains of course exact when considering it as an exact sequence of right $B$-modules. Moreover, if $P$ is a right projective $A$-module then it is also projective as a right $B$-module. Indeed, this is true for the free rank one $A$-module $B \oplus M$. Then the standard arguments enable to conclude.

To prove that if $B$ is smooth then so is $A$, we begin by proving that any induced $A$-module $Z=A \otimes_{B} Y$ is of finite projective dimension. Let $Q_{*} \rightarrow Y$ be a finite left $B$-projective resolution of $Y$. The functor $A \otimes_{B}-$ is exact since $A$ is right projective. Moreover if $Q$ is a left projective $B$-module, then $A \otimes_{B} Q$ is a left projective $A$-module, this follows from the fact that this is true for $Q=B$. Therefore $A \otimes_{B} Q_{*} \rightarrow A \otimes_{B} Y$ is a finite left $A$-projective resolution of the induced module $Z$.

Let $X$ be a left $A$-module. We claim that there exists an exact sequence of $A$ modules $0 \rightarrow Z_{n} \rightarrow Z_{n-1} \rightarrow \cdots \rightarrow Z_{0} \rightarrow X \rightarrow 0$ where the $A$-modules $Z_{i}$ are induced. This claim ends the proof, indeed each $Z_{i}$ is of finite projective dimension by the previous assertion, hence $X$ is of finite projective dimension.

To prove the claim, consider the relative reduced bar resolution 2.2. which is finite since $M$ is $B$-tensor nilpotent. Moreover its contracting homotopy is a right $A$-module map, see Remark 2.4. Consequently the relative reduced bar resolution remains exact by applying the functor $-\otimes_{A} X$. For some $n$ we obtain

$$
0 \rightarrow A \otimes_{B} M^{\otimes_{B} n} \otimes_{B} X \rightarrow \cdots \rightarrow A \otimes_{B} M \otimes_{B} X \rightarrow A \otimes_{B} X \rightarrow X \rightarrow 0 .
$$

Note that all the $A$-modules except $X$ are induced $A$-modules.

\section{References}

[1] Assem, I.; Simson, D.; Skowronski, A. Elements of the representation theory of associative algebras. Vol. 1. Techniques of representation theory. London Mathematical Society Student Texts, 65. Cambridge University Press, Cambridge, 2006. 
[2] Auslander, M. On the dimension of modules and algebras. III. Global dimension. Nagoya Math J. 9 (1955), 67-77.

[3] Auslander, M.; Buchsbaum, D. A. Homological dimension in Noetherian rings. Proc. Nat. Acad. Sci. U.S.A. 42 (1956) 36-38.

[4] Avramov, L. L.; Vigué-Poirrier M. Hochschild homology criteria for smoothness. Internat. Math. Res. Notices 1992, 17-25.

[5] Bergh, P. A.; Erdmann, K. Homology and cohomology of quantum complete intersections. Algebra Number Theory 2 (2008), 501-522.

[6] Bergh, P. A.; Madsen, D. Hochschild homology and global dimension. Bull. Lond. Math. Soc. 41 (2009), 473-482.

[7] Bergh, P. A.; Madsen, D. Hochschild homology and trivial extension algebras. Proc. Amer. Math. Soc. 145 (2017), 1475-1480.

[8] Buenos Aires Cyclic Homology Group. Cyclic homology of algebras with one generator.(J.A. Guccione, J. J. Guccione, M. J. Redondo, A. Solotar and O. Villamayor participated in this research) K-Theory 5 (1991), 51-69.

[9] Bühler, T. Exact categories, Expo. Math. 28 (2010), 1-69.

[10] Cartan, H.; Eilenberg, S. Homological algebra. Princeton University Press, Princeton, N. J., 1956.

[11] Cibils, C. Rigidity of truncated quiver algebras. Adv. Math. 79 (1990), 18-42.

[12] Cibils, C. Tensor Hochschild homology and cohomology. Interactions between ring theory and representations of algebras (Murcia), 35-51, Lecture Notes in Pure and Appl. Math., 210, Dekker, New York, 2000.

[13] Cibils, C.; Redondo, M. J.; Solotar, A. Han's conjecture and Hochschild homology for null-square projective algebras. To appear in Indiana University Mathematics Journal. arXiv:1703.02131/v2

[14] Cibils, C.; Lanzilotta, M.; Marcos, M.; Solotar, A. Adding or deleting arrows of a bound quiver algebra and Hochschild (co)homology. To appear in Proceedings of the American Mathematical Society.

DOI: https://doi.org/10.1090/proc/14936

[15] Gabriel, P. Unzerlegbare darstellungen I, Manuscripta Math. 6 (1972), 71-103.

[16] Gabriel, P. Indecomposable representations. II. Symposia Mathematica, Vol. XI (Convegno di Algebra Commutativa, INDAM, Rome, 1971), Academic Press, London, 1973.

[17] Gabriel, P. Auslander-Reiten sequences and representation-finite algebras. Representation theory, I (Proc. Workshop, Carleton Univ., Ottawa, Ont., 1979), Lecture Notes in Math., 831, Springer, Berlin, 1980.

[18] Gerstenhaber M.; Schack S. A Hodge-type decomposition for commutative algebra cohomology, J. Pure Appl. Algebra 48 (1987), 229-247. 
[19] Green E.L.; Psaroudakis, C.; Solberg, $\varnothing$. Reduction techniques for the finitistic dimension, arXiv:1808.03564

[20] Han, Y. Hochschild (co)homology dimension. J. London Math. Soc. 73 (2006), $657-668$.

[21] Hochschild, G. On the cohomology groups of an associative algebra, Ann. Math. 46 (1945), 58-67.

[22] Hochschild, G. Relative homological algebra, Trans. Amer. Math. Soc. 82 (1956), 246-269.

[23] lyengar, S. André-Quillen homology of commutative algebras. Interactions between homotopy theory and algebra, 203-234. Contemporary Mathematics, 436. American Mathematical Society, Providence, RI, 2007.

[24] Kaygun, A. Jacobi-Zariski exact sequence for Hochschild homology and cyclic (co)homology, Homology Homotopy Appl. 14 (2012), 65-78.

[25] Kaygun, A. Erratum to "Jacobi-Zariski exact sequence for Hochschild homology and cyclic (co)homology" Homology Homotopy Appl. 21 (2019), 301-303.

[26] McCleary, J. A user's guide to spectral sequences. Second edition. Cambridge Studies in Advanced Mathematics, 58. Cambridge University Press, Cambridge, 2001.

[27] Quillen, D. Higher algebraic K -theory. I, Algebraic K -theory, I: Higher K theories, Proceedings of the Conference, Battelle Memorial Institute, Seattle, Washington, 1972, Lecture Notes in Math., 341, Springer, Berlin, 1973.

[28] Ronco M. On the Hochschild homology decomposition, Comm. Algebra 21 (1993), 4694-4712.

[29] Schiffler, R. Quiver representations. CMS Books in Mathematics/Ouvrages de Mathématiques de la SMC. Springer, Cham, 2014.

[30] Serre, J.-P. Algèbre locale. Multiplicités. Lecture Notes in Math. 11. SpringerVerlag, Berlin, 1965.

[31] Solotar, A.; Suárez-Alvarez, M.; Vivas, Q. Hochschild homology and cohomology of generalized Weyl algebras: the quantum case. Ann. Inst. Fourier (Grenoble) 63 (2013), 923-956.

[32] Solotar, A.; Vigué-Poirrier M. Two classes of algebras with infinite Hochschild homology, Proc. Amer. Math. Soc. 138 (2010), 861-869.

[33] Vigué-Poirrier M., Décompositions de I'homologie cyclique des algèbres différentielles graduées commutatives, K-theory 4 (1991), 399-410.

[34] Weibel, C. A. An introduction to homological algebra. Cambridge Studies in Advanced Mathematics, 38. Cambridge University Press, Cambridge, 1994.

C.C.:

Institut Montpelliérain Alexander Grothendieck, CNRS, Univ. Montpellier, France.

Claude.Cibils@umontpellier.fr 
M.L.

Instituto de Matemática y Estadística "Rafael Laguardia", Facultad de Ingeniería, Universidad de la República, Uruguay.

marclan@fing.edu.uy

E.N.M.:

Departamento de Matemática, IME-USP, Universidade de São Paulo, Brazil.

enmarcos@ime.usp.br

A.S.:

IMAS-CONICET y Departamento de Matemática, Facultad de Ciencias Exactas y Naturales, Universidad de Buenos Aires, Argentina.

asolotar@dm. uba.ar 